\title{
Competition between Resonance Ejection and Ion Dissociation during Resonant Excitation in a Quadrupole Ion Trap
}

\author{
M. Judith Charles \\ Department of Environmental Sciences and Engineering, University of North Carolina at Chapel Hill. \\ Chapel Hill, North Carolina, USA
}

\author{
Scott A. McLuckey and Gary L. Glish* \\ Analytical Chemistry Division, Oak Ridge National Laboratory, Oak Ridge, Tennessee, USA
}

\begin{abstract}
The competition between ion dissociation and ion ejection during resonant excitation in a quadrupole ion trap is investigated. Ions of similar mass but with a range of critical energies for the onset of dissociation have been examined. The effects of the amplitude and duration of the resonant excitation, the well depth in which the ions are trapped, and the pressure and nature of the collision gas are explored. Once the onset of ion ejection is reached, the rate of ion ejection increases with increased amplitude of the resonant excitation signal. The rate of ejection decreases or stays constant as a function of the duration of the resonant excitation, depending upon the ion species being excited. Increasing the trapping well depth increases the relative amount of dissociation versus ejection as does increasing the pressure of the bath gas. Adding heavier bath gases lowers the onset of ion dissociation and raises the onset of ion ejection. ( $\mathrm{Am}$ Soc Mass Spectrom 1994, 5, 1031-1041)
\end{abstract}

$\mathrm{T}$ he quadrupole ion trap is becoming a valuable tool for performing tandem mass spectrometry (MS/MS) experiments to obtain structural information about organic molecules either by ion-molecule reactions [1] or collision-induced dissociation (CID) [2]. The most common means to effect CID in a quadrupole ion trap is to apply a radiofrequency ( $\mathrm{rf}$ ) signal in the dipolar mode to the endcap electrodes, which causes ions with that axial secular frequency motion to undergo resonant excitation and to gain kinetic energy in the axial direction [2]. This gain in kinetic energy can result in ion ejection or the kinetic energy of the resonantly excited ion can be converted into internal energy via collision with a target gas, thereby promoting ion dissociation and the formation of product ions. Thus, there are two possible results, which are competitive, when an ion undergoes resonant excitation. For an MS/MS experiment the spectrum obtained depends on which process dominates and is related to the rate power is absorbed by the parent ion and the frequency and efficiency of conversion of the resonant ion's kinetic energy to other modes (e.g., intemal energy) via collision.

Address reprint requests to $M$. Judith Charles, Department of Environmental Sciences and Engineering, University of North Carolina at Chapel Hill, Chapel Hill, NC 27599-7400.

* Current address: Department of Chemistry, University of North Carolina at Chapel I Iil], Chapel Hill, NC 27599-3290.
The parent ion absorbs power when its periodic motion is in resonance with the frequency of the rf voltage that is applied to the endcap electrodes. This power absorption has been investigated theoretically by trajectory calculations although these calculations did not include the effects of collisions [3, 4]. The magnitude of the power absorbed is directly related to the amplitude of the resonant excitation voltage, the duration that the resonant excitation voltage is applied, and the power lost by collisions with the target gas, according to the equation [5]

$$
A=\left(e^{2} E_{f}^{2} t / 8 m\right)+(d v / d t)_{\text {coll }}
$$

in which $A$ is the average power absorption, $e$ is the charge, $E_{f}$ is the amplitude of resonant excitation voltage (peak to peak), $t$ is the duration that the resonant excitation voltage is applied, $m$ is mass, and $(d t / d t)_{\text {coll }}$ is power loss by collisions.

The fraction of ions ejected from the ion trap versus the fraction that dissociate is, among other parameters, strongly dependent on the $q_{z}$ value. For $q_{z}<0.4$ the pseudopotential well model of ion motion (eq 2) shows that the maximum kinetic energy that an ion can have and remain trapped increases with $q_{z}^{2}$ :

$$
D_{z}=q_{z} V / 8=q_{z}^{2} m r^{2} \Omega^{2} / 32 e
$$


In this equation $D$ is the depth of well, $q_{z}$ is the Mathieu parameter, $V$ is the amplitude of the fundamental rf voltage (zero to peak), $e$ is the charge, and $r$ is the radius of the ion trap.

The pressure of the bath gas (generally helium) and the population of the ions can also affect ion ejection-dissociation. The bath gas serves two purposes. First, the general purpose of the bath gas is collisional damping of the trajectories of the ions, which improves resolution and sensitivity [6]. In the MS/MS experiment the bath gas also serves as the target gas for collisional activation. This latter process involves specifically converting an ion's kinetic energy to internal energy, whereas the former process just concerns decreasing an ion's kinetic energy.

Previous studies have demonstrated that operational parameters, which include the nature of the target gas, the target gas pressure, reaction time, and the $q_{z}$ value, have an effect on CID of ions in the ion trap mass spectrometer $[2,7]$. Information is lacking however with respect to the effect of these parameters on the average power absorbed by the parent ion. Specifically, little is known about the gain in kinetic energy that occurs when the ion absorbs power or the loss in kinetic energy that occurs when the ion converts its kinetic energy to internal energy, or to kinetic or internal excitation of the bath gas, during collisions with the gas. The experiments discussed herein were performed to gain further insight on the effect of the $q_{z}$ parameter, the amplitude of the resonant excitation voltage, the duration of the resonant excitation voltage, the composition of the bath gas, and the pressure of the bath gas on parent ion ejection-dissociation of four model ions of similar mass, but with different critical energies for dissociation. In contrast to previous studies, which generally focussed on one ion, this study helps to illustrate the contribution of the noninstrumental component to power absorption (eq 1).

\section{Experimental}

\section{Model Ions}

The effect of the experimental parameters on ion ejection was studied by using xenon and the effect of the experimental parameters on ion ejection-ion dissociation was studied by using ions generated from $n$ butylbenzene, perfluorotributylamine, and $N, N$-dimethylaniline (Aldrich Chemical Co., Milwaukee, WI). These compounds were chosen because they form ions that are similar in mass to the $m / z 132$ ion of xenon and because these ions vary in stability. (Experiments on a beam instrument show that the relative order of stability is $m / z 131$ from perfluorotributylamine $>$ $m / z 120$ from $N, N$-dimethylaniline $>m / z 134$ from $n$-butylbenzene.) The $m / z 134$ ion from $n$-butylbenzene dissociates to form product ions at $m / z 92$ and
91; the $m / z 131$ ion from perfluorotributylamine dissociates to form a product ion at $m / z 69$. Dissociation of the $m / z 120$ ion from $N, N$-diethylamine produces products at $m / z 118,103,93,91,79$, and 77 . The $m / z$ $120[\mathrm{M}-\mathrm{H}]^{+}$ion was chosen over the $\mathrm{M}^{+\cdot}$ ion because the latter dissociates exclusively by loss of $\mathrm{H}^{\circ}$ whereas the former gives a number of product ions. The product ions from $m / z 120$ represent various competitive dissociations that involve rearrangements or simple cleavage and thus should provide extra insight into the kinetics of CID in the ion trap.

\section{MS/MS Experiments}

All experiments were performed on a Finnigan ion trap mass spectrometer (Finnigan-MAT, San Jose, CA). Analytes were introduced into the ion trap through a batch inlet system. Sample pressures were typically in the $10^{-7}$-torr range or less. Experiments were performed to make sure that charge exchange or other types of reactions of the ions of interest with the neutral analyte did not contribute to the observed results at the pressures used (e.g., no other ions from other $X_{e}$ isotopes were observed, which demonstrates that self-charge-exchange does not occur to an appreciable extent under these experimental conditions). A helium pressure of $7.0 \times 10^{-4}$ torr was used unless noted otherwise. The mode of ionization was electron ionization and an electron pulse of $1 \mathrm{~ms}$ was typically employed. The parent ion was isolated by ramping the radiofrequency voltage on the ring electrode (mass selective instability mode) to eject ions with mass-tocharge ratio values less than the parent ion. In instances in which ions with mass-to-charge ratio values greater than the parent ion were present, these ions were resonantly ejected by applying a supplementary If voltage to the endcap electrodes during the rf ramp. The resonant excitation frequency on the endcap electrodes was adjusted to obtain optimum resonance between the parent ion and the applied voltage. The resonant excitation voltage (peak-to-peak amplitudes) was applied to the endcap electrodes in the dipolar mode for $5,10,20,30$, or $50 \mathrm{~ms}$ in the presence and absence of helium at a $q_{z}$ value of $0.1,0.2$, or 0.3 . The experiments for a particular ion were performed consecutively. If necessary, the ionization time was adjusted between experiments to maintain similar ion populations.

Investigation of the effect of helium pressure on ion ejection-dissociation of perfluorotributylamine and $N, N$-dimethylaniline was performed by altering the helium flow to obtain pressures of $3.5 \times 10^{-4}, 7.0 \times$ $10^{-4}$, and $3.5 \times 10^{-3}$ torr. The ionization time used in these experiments was modified to minimize differences among the ion populations at each pressure. Gases other than helium ( $\mathrm{Ar}, \mathrm{Xe}$ ) also were used to study the effect of the composition of the bath gas. 


\section{Results and Discussion}

Ion ejection of the $m / z 132$ ion of xenon. In the absence of helium, the onset of ion ejection for $m / z 132$ of xenon occurs at the same resonant excitation voltage $(30 \mathrm{mV})$ at $q_{z}=0.1,0.2$, and 0.3 . The percentage of the ion population that is ejected at a specified resonant excitation voltage however is dependent on the $q_{z}$ value. The voltage required to eject $50 \%$ of the jons with a 20-ms resonant excitation period was $150 \mathrm{mV}$ at $q_{z}=0.3,100 \mathrm{mV}$ at $q_{z}=0.2$, and $50 \mathrm{mV}$ at $q_{z}=0.1$ (Figure 1a). If we assume that the voltage required to eject $50 \%$ of the ions is a measure of the average kinetic energy, then this result would be expected if eq 1 holds for the ion trap. The power absorbance is the change in energy per change in time, and integration of eq 1 with respect to time shows that, with all other parameters constant, the energy should be proportional to the square of the resonance excitation amplitude. Because the well depth is proportional to the square of the $q_{z}$ value (eq 2), it is expected that the $q_{z}$ value should be linearly related to the voltage required for resonant ejection, as is observed.

The presence of helium does not appear to have an effect on the resonant excitation voltage needed to initiate ion ejection or the degree of ion ejection at $q_{z}=0.1$ (50\% of the ions are ejected at $50 \mathrm{mV}$ ) and has only a slight effect at $q_{z}=0.2$. At $q_{z}=0.3$, however, the presence of helium obviously affects the ion ejec-

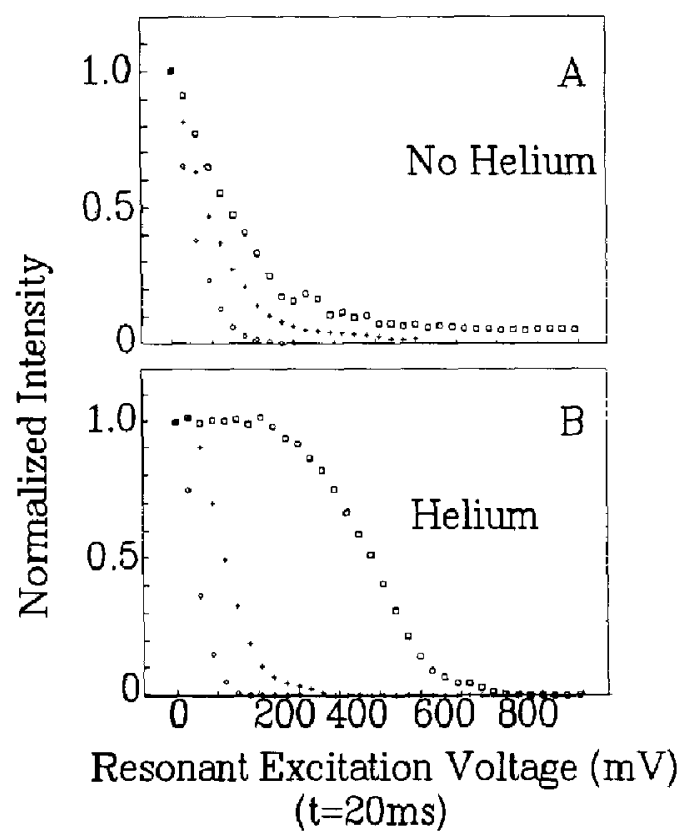

Figure 1. The effect of $q_{z}$ value on ion ejection of xenon $(\mathrm{m} / \mathrm{z}$ 132 ) in the presence and absence of helium (the resonant excitation voltage was applied for $20 \mathrm{~ms}$ ). $\square: q_{2}=0.3 ;+: q_{z}=0.2 ; \varnothing$; $q_{z}=0.1$. tion process. Under these conditions, the onset of ion ejection does not occur until the resonant excitation signal is $240 \mathrm{mV}$ and $50 \%$ of the ions are ejected at 480 $\mathrm{mV}$ in the presence of helium. This result indicates that at $q_{z}=0.3$ collisions with helium begin to cool efficiently the ions' kinetic energy, which results in the need for a greater resonant excitation amplitude for ion ejection. Under these conditions the well depth for $m / z 132$ is $18.9 \mathrm{eV}$, which means the maximum relative collision energy of this ion with helium is slightly over $0.5 \mathrm{eV}$. Therefore, the only mechanism for kinetic cooling is transfer of ion kinetic energy to the target ( $\mathrm{He}$ ). The more effective kinetic cooling of the ions at $q_{z}=0.3$ can be attributed to the greater number of collisions that occur at the greater trapping well depth. The deeper the trapping well, the longer it will take the ions to be resonantly excited to the energy necessary for ejection and therefore they will have a longer path length and undergo more collisions.

Over the range of resonant excitation times studied (5-50 ms) the duration that the resonant excitation amplitude is applied has little effect on ion ejection for xenon ions (Figure 2). Integrating the first part of eq 1 with respect to time indicates that the kinetic energy gain of the ion from the resonant excitation voltage should be proportional to the square of the resonant excitation time in the absence of a mechanism for power dissipation. The power dissipation via collisions with $\mathrm{He}$ increases with ion energy. At some point, therefore, energy gain and energy loss should balance. If this is the only effect, then the Xe ions rapidly $(<5$ ms) reach this steady state of energy gain-loss. However, an additional factor must be taken into consideration in the interpretation of Figure 2. The Finnigan ion trap is not an ideal quadrupole geometry; it is elongated in the $z$-direction $[8,9]$ and this elongation introduces higher order nonlinear trapping fields. It has been shown theoretically that the resonant frequency of the ions changes as the kinetic energy of the ions changes [10]. Thus, as the ion increases its kinetic energy from power absorption, its resonant frequency will shift and the power absorption will become less and less efficient. The former effect, in which acceleration and deceleration are balanced, is expected to occur even if there are no ion frequency shifts. However, the role that the latter effect plays in determining the point at which ion ejection becomes time independent cannot be determined from these results.

Ion ejection-dissociation in the absence of helium. Without helium as a damping and collision gas, ion ejection dominates over ion dissociation for all the polyatomic ions studied. The predominance of ion ejection is evident because the level of $\Sigma_{i}$ and the parent ion signal decrease essentially in unison. Some dissociation does occur and the magnitude of this process is dependent on the compound and the $q_{z}$ value. For $N, N$-dimethylaniline and perfluorotributylamine, the parent ion dis- 


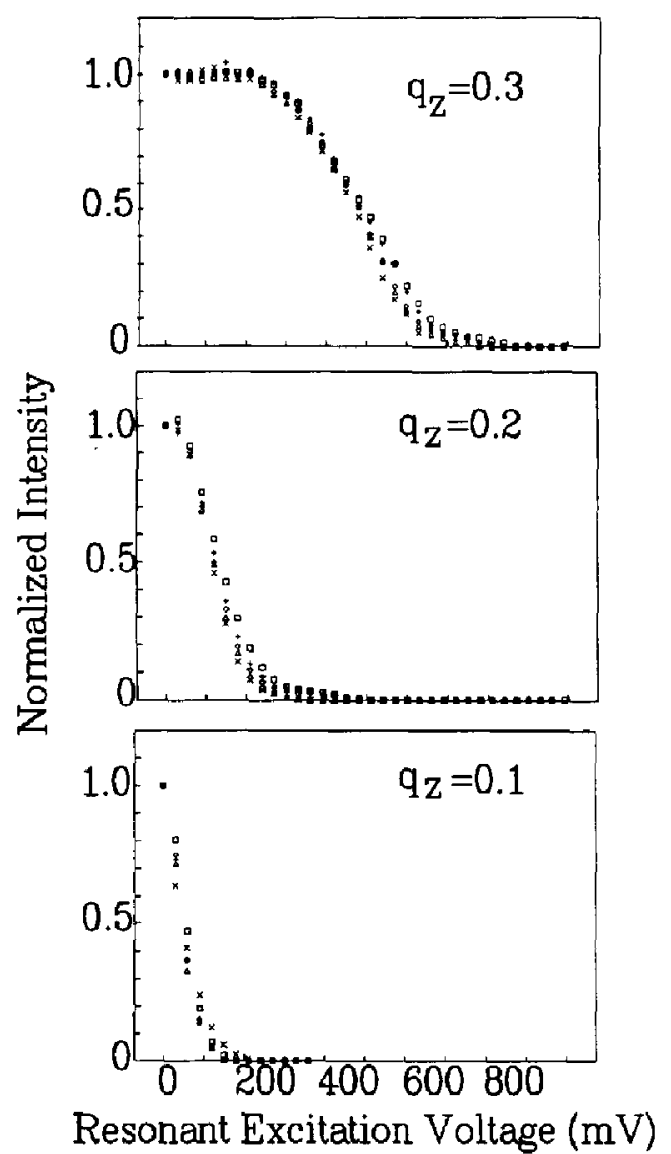

Figure 2. The effect of $q_{z}$ value and the duration that the resonant excitation voltage is applied on ion ejection of xenon $(\mathrm{m} / z \mathrm{132})$ in the presence of helium. Time: $\square: 5 \mathrm{~ms}$; $: \mathbf{1 0} \mathrm{ms}$; o: $20 \mathrm{~ms} ; \Delta: 30 \mathrm{~ms} ; \mathrm{x}: 50 \mathrm{~ms}$.

sociation is observed at $q_{x}=0.2$ and 0.3 . The product ions that are formed comprise less than 5 and $1 \%$ of the total ion current of $N, N$-dimethylaniline and perfluorotributylamine, respectively. Dissociation of the butylbenzene molecular ion occurs at $q_{z}=0.1,0.2$, and 0.3 . Product ions formed under these conditions constitute less than $20 \%$ of the total ion current. For all the compounds, the resonant excitation voltage at which ion ejection begins is independent of $q_{z}$ value, but greater voltages are needed to eject $50 \%$ of the ions when the $q_{z}$ value is increased. The reason for this occurrence, as previously stated, is because the kinetic energy needed to eject an ion increases with the well depth. The dissociation that occurs without a bath gas is most likely a result of collisions of the ion with neutral analyte and other background gases.

Ion ejection-dissociation in the presence of helium. Three observations can be made about ion dissociation-ion ejection for the compounds in the presence versus absence of helium. First, the resonant excitation volt- age that is needed to initiate ion ejection-dissociation increases in the presence of helium and increases as the $q_{z}$ value is increased. In the absence of helium, the onset of ion ejection occurs at a resonant excitation voltage of $30 \mathrm{mV}$ at $q_{z}=0.1,0.2$, and 0.3 . In comparison, in the presence of helium ion ejection-dissociation occurs at $60 \mathrm{mV}$ at $q_{z}=0.1,90 \mathrm{mV}$ at $q_{z}=0.2$, and at $150 \mathrm{mV}$ at $q_{2}=0.3$. Second, ion ejection is the predominant process both in the absence and presence of helium at $q_{z}=0.1$. Third, the relative and absolute degree of ion dissociation is enhanced as the $q_{z}$ value is increased for all the compounds in the presence of helium. However, the balance between ion ejection and ion dissociation and the effect of the duration of the resonant excitation voltage differ among the model ions.

At $q_{z}=0.2$, the onsel of ion dissociation for $N, N-$ dimethylaniline occurs at $90 \mathrm{mV}$ when the resonant excitation voltage is applied for $20 \mathrm{~ms}$ (Figure 3). At this resonant excitation voltage, ion dissociation and ion ejection both occur (a decrease in $\Sigma_{i}$ and the parent

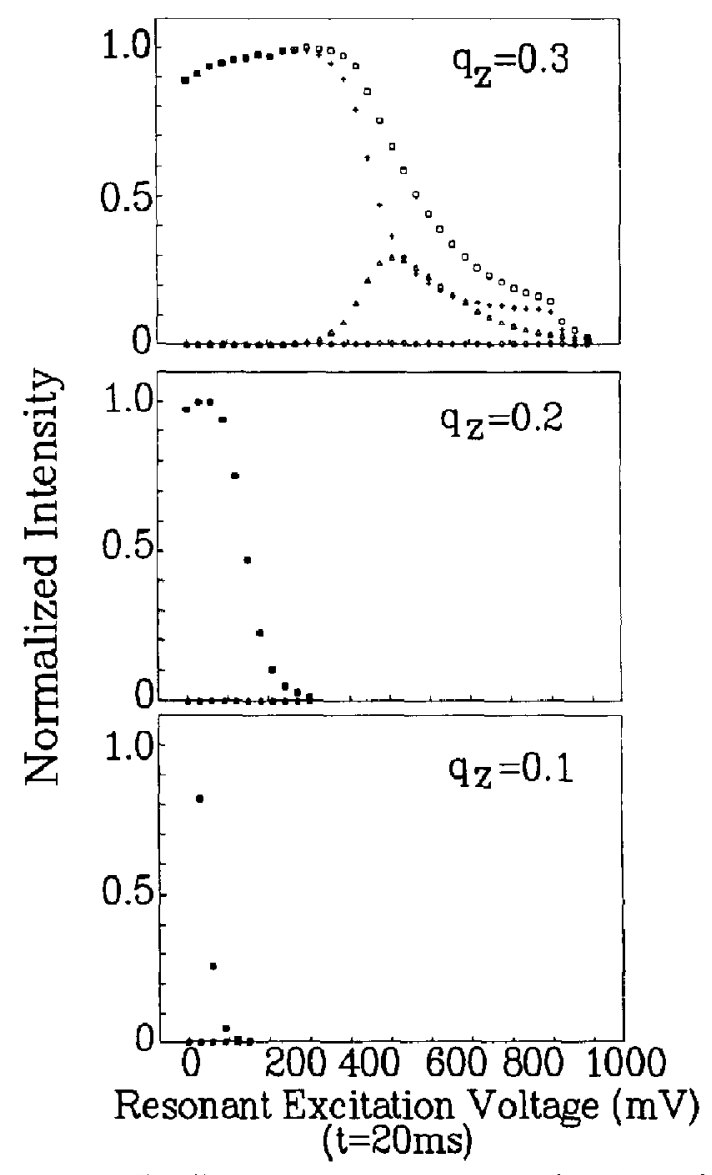

Figure 3. The effect of $q_{z}$ value on ion ejection-dissociation of $N, N$-dimethylaniline $(m / z 120)$ in the presence of helium (the resonant excitation voltage was applied for $20 \mathrm{~ms}$ ). $\square$ : $\Sigma_{i}$; + : $m / z 131 ; \diamond: m / z 119 ; \Delta: m / z 69$. 


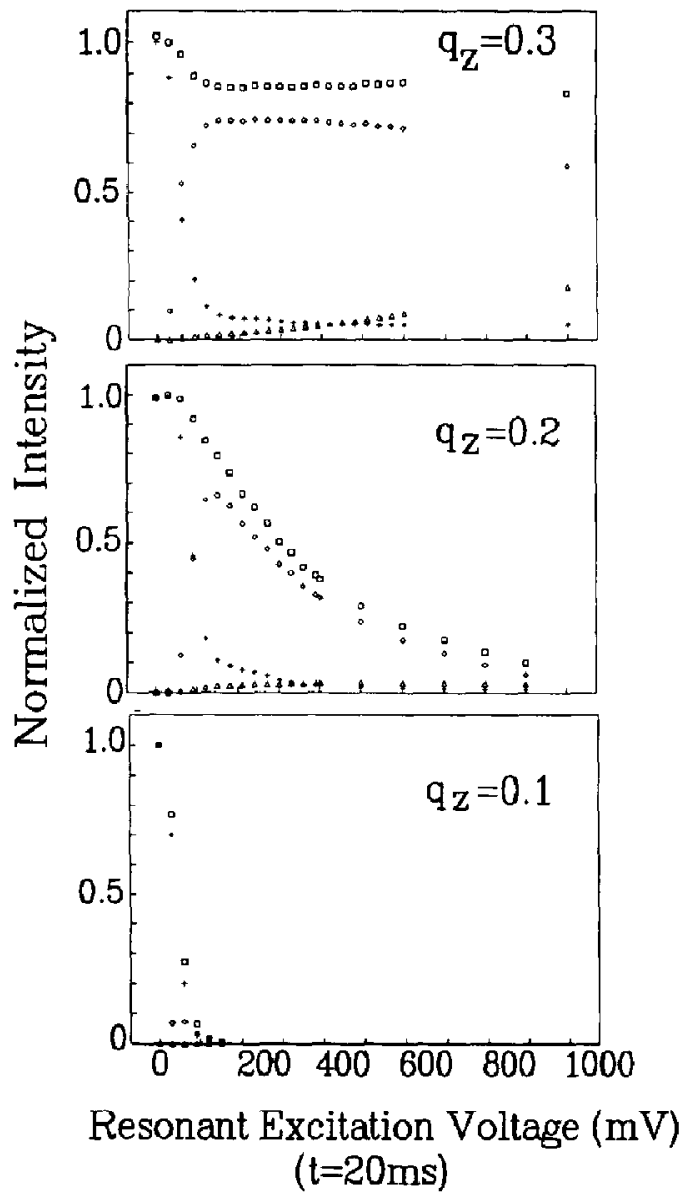

Figure 4. The effect of $q_{2}$ value ion ejection-dissociation of butylbenzene $(m / z 134)$ in the presence of helium (the resonant excitation voltage was applied for $20 \mathrm{~ms}$. $\square: \Sigma_{i ;}+: m / z$ 134; 0 : $m / z 92 ; \Delta: m / z 91$.

ion intensity and an increase in the intensity of the product ions is observed). At $a_{7}=0.3$, ion dissociation and ion ejection also both occur with the rate of dissociation increasing relative to the rate of ejection as evidenced by an increase in the formation of the product ions in comparison to $q_{z}=0.2$ (a maximum of $24 \%$ of the ion current in the most abundant product ion at $q_{2}=0.3$, compared to $10 \%$ at $q_{z}=0.2$ ). Again we observe that the resonant excitation voltage needed for the onset and completion of ion ejection-dissociation increases as the $q_{z}$ value is increased.

Ion dissociation of butylbenzene occurs at $q_{z}=0.1$, 0.2 , and 0.3 (Figure 4). Dissociation occurs competitively with ion ejection at $q_{z}=0.1$ and 0.2 , but predominates at $q_{z}=0.3$. At $q_{z}=0.1$, the intensity of the $m / z 92$ product ion is less than $10 \%$ of the total ion current and all the ions are ejected from the trap when $150 \mathrm{mV}$ are applied. The onset of ion ejection-dissociation occurs simultaneously at $q_{z}=0.2$, as it does at $q_{z}=0.1$. At $q_{z}=0.2$ the intensity of the $m / z 92$ prod-
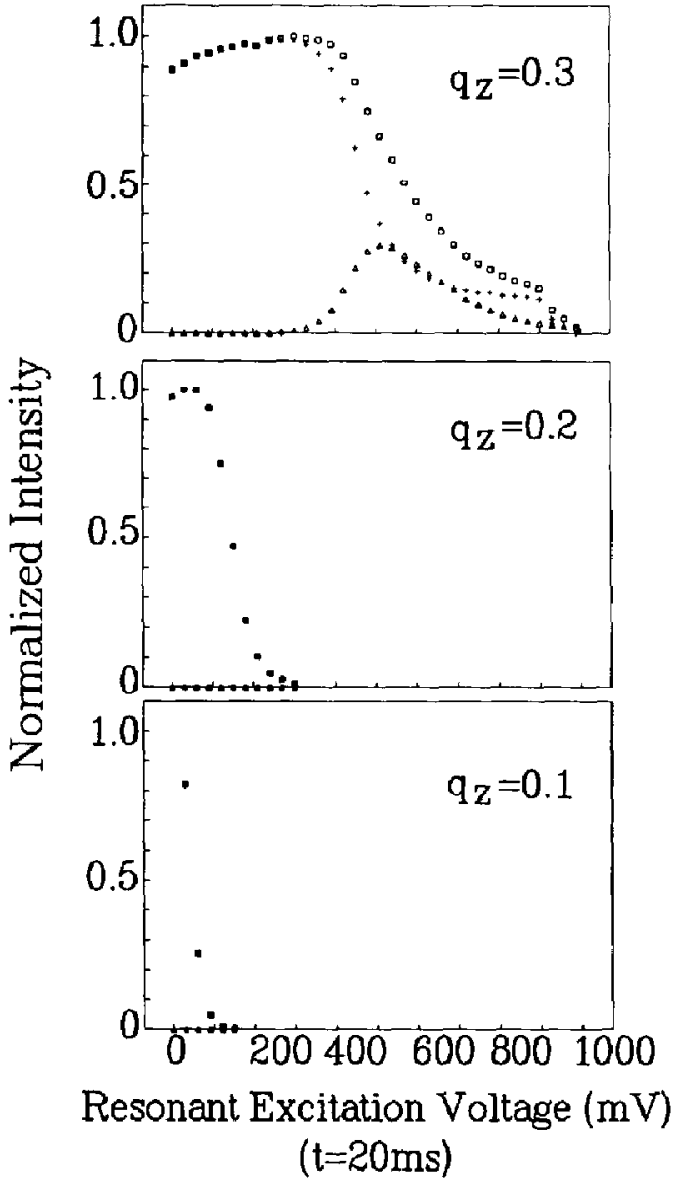

Figure 5. The effect of $q_{z}$ value on ion ejection-dissociation of perfluorotributylamine $(m / z, 131)$ in the presence of helium (the resonant excitation voltage was applied for $20 \mathrm{~ms}$ ). $\square: \Sigma_{i} ;+$ : $m / z 131 ; \Delta: m / 269$.

uct ion reaches $64 \%$ at $120 \mathrm{mV}$. At $q_{z}=0.3$, ion dissociation dominates from a resonant excitation amplitude of $30 \mathrm{mV}$ to greater than $1000 \mathrm{mV}$.

Ion dissociation of $m / z \mathbf{1 3 1}$ from perfluorotributylamine is not observed until $q_{z}=0.3$. The onset of ion dissociation occurs at $300 \mathrm{mV}$ and ion dissociation predominates until a resonant excitation voltage of 420 $\mathrm{mV}$ is applied to the endcap electrodes (the level of $\Sigma_{i}$ remains constant while the intensity of the $\mathrm{m} / z 131$ parent ion decreases and the intensity of the $m / z 69$ product ion increases; Figure 5). The normalized intensity of the product ion at $m / z 69$ reaches a maximum value of $30 \%$ at a resonant excitation voltage of 510 $\mathrm{mV}$.

A summary of the differences in the behavior of the four model ions under similar conditions (a resonant excitation voltage of $20 \mathrm{~ms}$ at $q_{z}=0.3$ ) is presented in Figure 6 . The decrease in the normalized intensity of the $m / z 132$ ion of xenon represents the situation in which only ion ejection can result due to resonant 


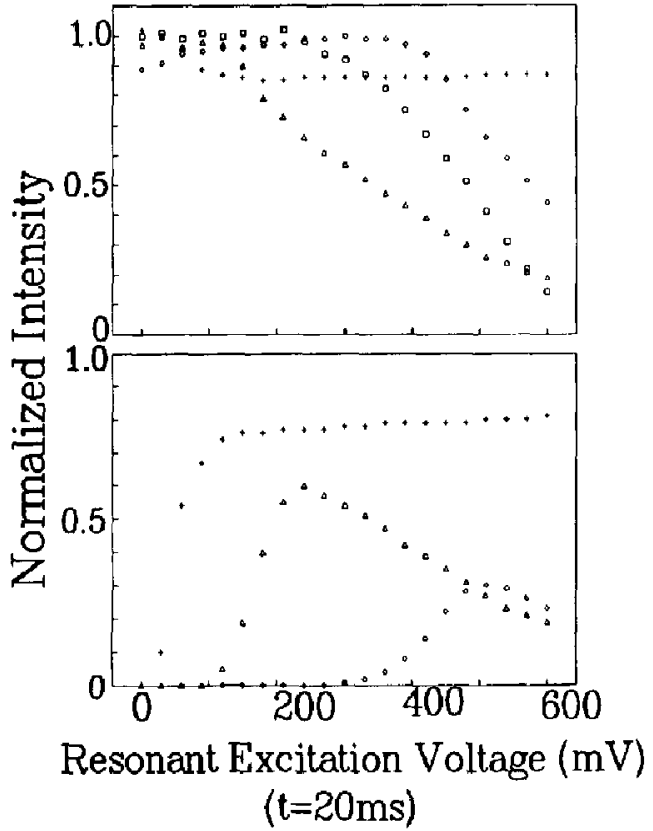

Figure 6. A comparison of ion ejection-dissociation of four model ions (the resonant excitation voltage was applied for 20 ms at $q_{z}=0.3$ ). Top: $\square$ : xenon; + : TIC butylbenzene; $\diamond$ : TIC perfluorotributylamine; $\triangle:$ TIC N,N-dimethylaniline. Bottom: + : $\Sigma$ products of butylbenzine; $\diamond$; product of perfluorotributylamine; $\Delta: \Sigma$ products of dimethylaniline.

excitation. In the presence of helium, at $q_{z}=0.3$ the kinetic energy of xenon is lowered by collisions with helium and a resonant excitation voltage of $240 \mathrm{mV}$ is needed to cause the $m / z 132$ ion to be ejected from the trap. Ejection of $n$-butylbenzene and perfluorotributylamine ions does not start to occur until higher resonant excitation amplitudes are applied. This is a result of the fact that these ions can transfer kinetic energy to internal energy by inelastic collisions with helium as well as undergoing elastic collisions with $\mathrm{He}$. The resonant excitation amplitude necessary to eject these ions from the ion trap depends on the efficiency of the conversion of kinetic energy to internal energy or to helium kinetic energy in the collisions with helium, and the critical energy for dissociation. Once a parent ion dissociates, the product ion is not resonantly excited and therefore will not be ejected from the ion trap. For $\Sigma_{i}$ to decrease, the parent ion must be ejected from the ion trap before it dissociates. Thus, for ions with similar efficiencies of converting kinetic energy to internal energy, the ion with the lower critical energy for dissociation will require a higher amplitude resonant excitation voltage to be ejected before it dissociates. In our experiments this is observed with the ions from butylbenzene requiring a greater resonant amplitude for ejection than perfluorotributylamine.

$N, N$-dimethylaniline deviates from what is expected in that the onset of ejection occurs prior to that for xenon. There are several factors that contribute to this deviation. First, this ion is approximately $90 \%$ the mass of Xe, so it will be in a shallower potential well. Because the power absorption from the resonant voltage is proportional to the square of the amplitude, the well depth will be about $80 \%$ of that for Xe; therefore it is expected that about $20 \%$ less voltage should be required to eject the ion from $N, N$-dimethylaniline if it loses power at a similar rate to the xenon ion. Experimentally even less than $80 \%$ of the resonant excitation voltage needed to eject xenon ions is required to eject the ions from $N, N$-dimethylaniline. Two other factors can be considered. One of these is that $m / z 120$ undergoes ion-molecule reactions with neutral $N, N$ dimethylaniline to produce ions at $m / z 121$ and 122 [11]. Also, because the ion loss occurs at approximately the onset of dissociation, ion loss associated with the dissociation may be occurring. This will occur in cases in which there is formation of a product ion with a $q_{z}$ value close to or greater than 0.91 . For $m / z 120$ from $N, N$-dimethylaniline, a small amount of product ion is observed at $m / z 42$. This is near the limit for stability and it is likely that this product ion is not trapped efficiently. Additionally, because $m / z 103$ is formed by loss of $\mathrm{NH}_{3}$ [11], formation of $\mathrm{NH}_{3}^{+\cdot}$ or $\mathrm{NH}_{4}^{+}$is a possibility, and neither of these ions would be trapped under the experimental conditions. (The main reason why the lowest reasonable value of $q_{z}$ should be used for MS/MS is to limit such losses of low mass product ions.)

The dissociation of the different ions is plotted in Figure 6b. The critical energy for dissociation of $\mathrm{m} / \mathrm{z}$ 134 of $n$-butylbenzene is less than the critical energy for dissociation of $m / z 120$ from $N, N$-dimethylaniline, and this latter parent ion has a lower critical energy for dissociation than $m / z \quad 131$ from perfluorotributylamine. As would be expected, this order of onset of dissociation is observed in the ion trap for ion dissociation as a function of resonant excitation amplitude. The onset for formation of butylbenzene product ions is at a lower resonant excitation voltage than the onset for the product ions of the $N, N$-dimethylaniline parent ion. The onset of formation of product ions from $\mathrm{m} / \mathrm{z}$ 131 from perfluorotributylamine is at a higher resonant excitation voltage than the other systems. Another influence of the relative critical energies also can be seen in Figure 6b. There is an inverse relationship between onset of ion dissociation and the CID efficiency. This is a result of the fact that lower critical energy dissociations can be accessed with lower energy collisional activation. As more or higher energy collisions are necessary to induce dissociation, it becomes more likely that an ion will be ejected from the ion trap before it undergoes the necessary collision(s) to promote dissociation.

By looking at both parts of Figure 6 (and Figures 3. 4 , and 5) another observation can be made. At the maximum CID efficiency for $n$-butylbenzene and $N, N$-dimethylaniline, the ion current consists entirely 
of product ions. All the parent ions that have not been ejected from the ion trap have dissociated and the subsequent decline in product ion current results from an increased rate of parent ion ejection relative to dissociation.

Effect of duration of the resonant excitation voltage. Differences also were observed among the compounds regarding the effect on ion ejection-dissociation with the duration that the resonant excitation voltage was applied to the endcap electrodes. For $N, N$-dimethylaniline, no effect on ion dissociation as a function of the duration of the resonant excitation was observed over the time range investigated. The absence of an effect of resonant excitation duration suggests that this parent ion from $N, N$-dimethylaniline very quickly reaches a steady state in which the rates of gain and loss of internal energy are equivalent. Thus, increasing the duration of resonant excitation does not affect the distribution of ions that dissociate versus ions that are ejected.

In contrast, the resonant excitation voltage required to initiate ion dissociation of $m / z 131$ from perfluorotributylamine decreases from about 400 to $240 \mathrm{mV}$ when the duration of the amplitude is increased from 10 to $50 \mathrm{~ms}$ (Figure 7). An increase in the maximum normalized intensity of the $m / z 69$ product ion (11 to $50 \%$ ) is also observed. At $10 \mathrm{~ms}$, ion ejection and dissociation proceed over a range of $110 \mathrm{mV}(400-510$ $\mathrm{mV}$ ). Ion ejection is the dominant process over the whole range of resonant excitation voltages at $10 \mathrm{~ms}$. At $50 \mathrm{~ms}$, ion dissociation begins at $240 \mathrm{mV}$ and predominates to $330 \mathrm{mV}$. From 330 to $480 \mathrm{mV}$ ion

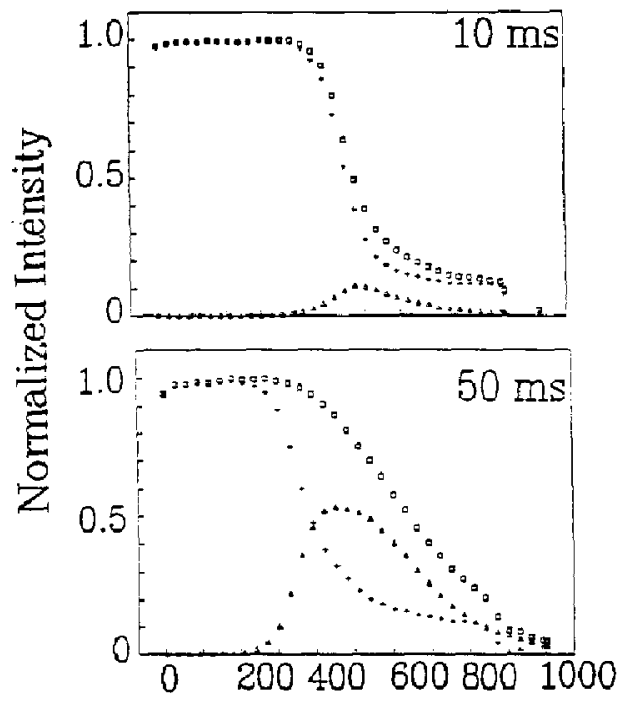

\section{Resonant Excitation Voltage (mV)}

Figure 7. The effect of the duration that the resonant excitation voltage is applied on ion ejection-dissociation of perfluorotributylamine $\left(g_{z}=0.3\right) . \square: \Sigma_{i}+: m / z 131 ; \Delta: m / z 69$. ejection becomes increasingly competitive with dissociation. Ion ejection predominates when greater resonant excitation voltages are employed. This suggests that the $m / z 131$ ion from perfluorotributylamine does not reach a steady state quickly, but continues to gain internal energy via collisions with He faster than it loses energy. Therefore, the use of lower resonant excitation voltages for longer durations will increase the fraction of ions that dissociate versus the fraction that are ejected.

Effect of helium pressure. The results for $m / z 131$ from perfluorotributylamine and $m / z 120$ from $N, N$ dimethylaniline are representative for the effects of the helium pressure (Figures 8 and 9, respectively). As the pressure is increased, the collision rate will increase. Thus, the second term in eq 1 will become more important and the end result will be that at higher pressures, dissociation will be favored increasingly versus ejection. This is evidenced in two ways in

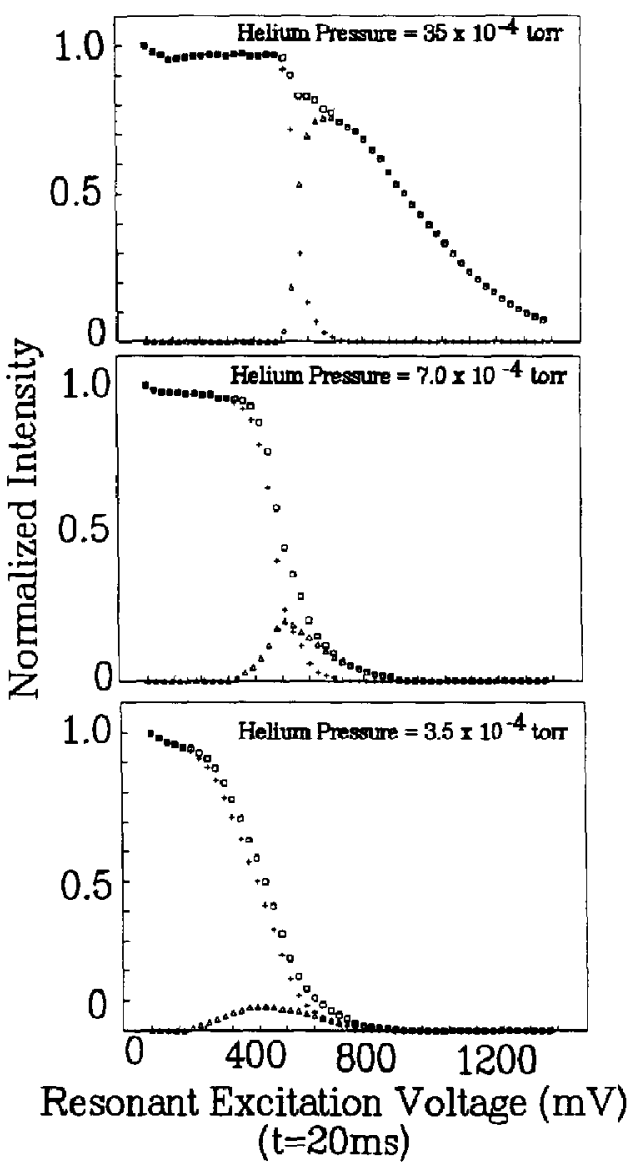

Figure 8. Plots of the dissociation of $m / z 131$ from perfluorotributylamine as a function of resonant excitation voltage for different pressures of the helium bath gas. $\square: \Sigma_{i} ;+: m / z$ 131; $\Delta: m / z 69$. 


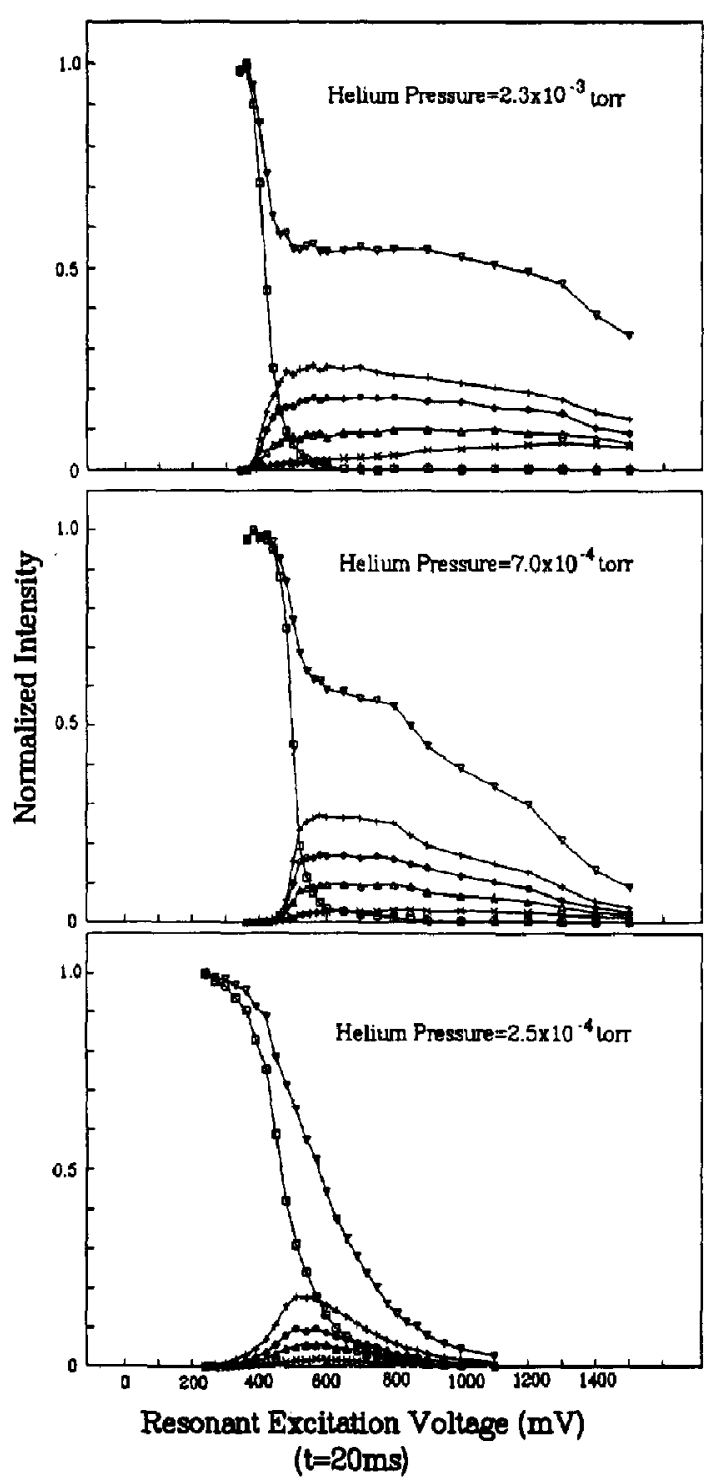

Figure 9. Plots of the parent $(m / z 120$ from $N, N$-dimethylaniline; open symbols) and product ion currents (solid symbols) and their sum as a function of resonant excitation voltage for different pressures of the helium bath gas. $\nabla: \Sigma_{i} ; \square: m / z$ 120; $+: m / z$ 118; $\Delta: m / z$ 103; $\diamond: m / z$ 91; X: $m / z \pi 7$.

Figures 8 and 9. First, the rate of decrease of the parent ion increases with increasing helium pressure. Second, higher resonant excitation voltages are required to eject ions as the helium pressure increases.

It also can be seen from Figure 8 that for $m / z 131$ the MS/MS efficiency (the sum of the product ions divided by the initial parent ion intensity [12]) increases with helium pressure. This results from the ion being able to attain a greater degree of internal excitation prior to ejection, thus increasing its probability of dissociation. For the $N, N$-dimethylaniline (Figure 9) the maximum MS/MS efficiency is obtained at a helium pressure of $7.0 \times 10^{-4}$ torr. Therefore the efficiency does not increase any at higher pressures. Finally, it can be noted that there is a decrease in the value for the onset of dissociation at lower helium pressures. Because the ions gain mote energy (absorb more power from the resonant voltage) between collisions at lower pressures there is a higher probability of higher energy collisions at a given resonant excitation amplitude, even though the overall collision probability is lower. For $m / z 131$ (Figure 8) the dissociation onset occurs at a resonant excitation amplitude of 180 $\mathrm{mV}$ at $3.5 \times 10^{-4}$ torr, at $330 \mathrm{mV}$ at $7.0 \times 10^{-4}$ torr, and at $510 \mathrm{mV}$ at $3.5 \times 10^{-3}$ torr. For N,N-dimethylaniline there is much less of a change in the resonant excitation voltage for onset of dissociation and actually it is essentially the same at the two higher pressures in Figure 9. The smaller difference is probably related to the lower critical energy for dissociation. The lack of change between the higher two helium pressures is likely to be due to the fact that conditions are at a point at which maximum MS/MS efficiency is achieved.

A final observation pertaining to the effects of helium pressure concerns sensitivity. The data in Figures 8 and 9 are plotted as relative intensities and normalized for each plot; however, the absolute ion current changes with pressure. For $N, N$-dimethylaniline, the maximum ion current is observed at $7.0 \times 10^{-4}$ torr. At $2.5 \times 10^{4}$-torr pressure the total ion signal is about a factor of 6 lower than at $7.0 \times 10^{-4}$. This is a result of less efficient initial damping of the ions at lower helium pressure. On the other end of the pressure range, $2.5 \times 10^{-3}$, the total ion signal is a factor of 2 less than at $7.0 \times 10^{4}$. This likely results from scattering loss of ions during the ejection to the multiplier. These ions have been shown to have kinetic energies in the range of hundreds to thousands of electrovolts [13], and thus have a greater probability of being scattered out of the acceptance of the multiplier at higher helium pressures.

Effect of heavier target gases. Helium is traditionally used as the target gas for MS/MS in the ion trap because it is the damping gas. Heavier gases are poorer damping gases because they will effect greater scattering of the ions, which results in ions being lost from the trap, reducing sensitivity [14]. However, greater energy deposition can be achieved with heavier target gases $[15,16]$. The amount of energy that potentially can be converted from kinetic to internal, to induce dissociation, is related to the mass of the collision gas:

$$
E_{\text {rel }}=E_{\text {lab }}\left[m_{i} /\left(m_{t}+m_{i}\right)\right]
$$

$E_{\text {rel }}$ is the maximum amount of kinetic energy that can be converted to internal energy, $E_{\text {lab }}$ is the laboratory collision energy, $m_{t}$ is the mass of the collision gas, and $m_{i}$ is the mass of the parent ion. Thus, for helium with the parent ions used in this study a maximum of 
about $3 \%$ of the laboratory collision energy can be converted to internal energy on any single collision. If argon is used as the collision gas, then $E_{\text {rel }}=23 \% E_{\text {lab; }}$; for xenon, $E_{\text {rel }}=50 \% E_{\text {lab }}$.

To investigate the use of heavier collision gases, the $N, N$-dimethylaniline system was chosen because it has a number of different dissociation pathways, some via complex rearrangements and others via direct cleavage. All the experiments were done with the heavier gas (argon or xenon) added to the system and the normal helium gas pressure maintained, because it was found that without the helium no ions were detected. Figure 10 shows the parent ion intensity and $z_{i}$ for two pressures of argon added to the helium, normalized to the results obtained by using just helium. It can be seen from this plot that there is loss of ions when argon is added to the system. This could be either from scattering during trapping or scattering during ion ejection to the detector.

The addition of $1 \times 10^{-5}$ torr of argon rhanges the overall trend very little. The onset of dissociation is the same and the subsequent $\Sigma_{i}$ decrease similarly. This is in contrast to adding $5 \times 10^{-5}$ torr of argon, in which case the dissociation onset is at a much lower voltage and competitive with ion ejection over a much wider range of voltages. These observations can be explained by the fact that even though most of the collisions of the ion are still going to be with helium, there will be approximately five times more collisions with argon at the higher pressure than at the lower pressure. Because collisions with argon can convert up to almost eight times more kinetic energy into internal energy, there is a greater probability relative to resonant ejection that an ion will be collisionally activated to sufficient internal energy for dissociation to occur. Another outcome of the use of a heavier gas and being able to use, more efficiently, higher resonant excitation voltages, is that

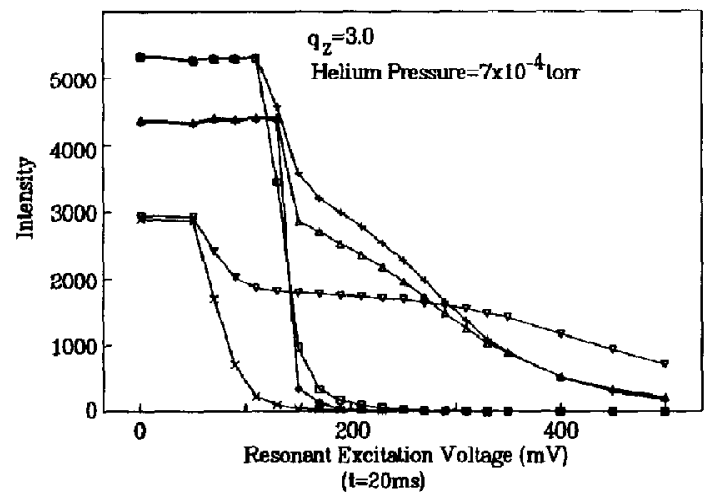

Figure 10. Plots of the parent ion current and sum of parent ion and the product ions shown in Figure 11, as a function of resonant excitation amplitude for various amounts of argon added to the system. All plots are normalized to the experiment in which to argon is added. + : $\Sigma_{i}$, no $\operatorname{argon;} \square: m / z 120$, no argon; $\Delta: \Sigma_{i}, 1 \times 10^{-5}$ torr argon; $O: m / z 120,1 \times 10^{-5}$ torr argon; $\nabla: \Sigma_{i}, 5 \times 10^{-5}$ torr argon; $X: m / z 120,5 \times 10^{-5}$ torr argon. there can be a variation in the relative dissociation channels. This is shown in Figure 11, which plots the relative intensity at each amplitude from Figure 10. It can be seen that the simple cleavage processes leading to $m / z 77$ and 105 increase much more in relative abundance with resonant excitation voltage when argon is present. Because simple cleavages typically have higher critical energies and more steeply rising rates as a function of internal energy than rearrangements, this result indicates that higher internal energies are achieved, as would be expected.

Collisional activation in the ion trap is a multistep process. Adding a heavier gas to the ion trap therefore decreases the number of collisions necessary to induce dissociation because a collision with argon can be the equivalent of eight collisions with helium. However, when considering the relative rates of competitive dissociation, only the last collision (i.e., the collision that increases the internal energy above that necessary for dissociation in the ion trap) is important. If this last collision is with argon, the higher energy pathways (e.g., simple cleavages) will be more competitive because the ion will have more excess internal energy than if the collision was with helium. This is even

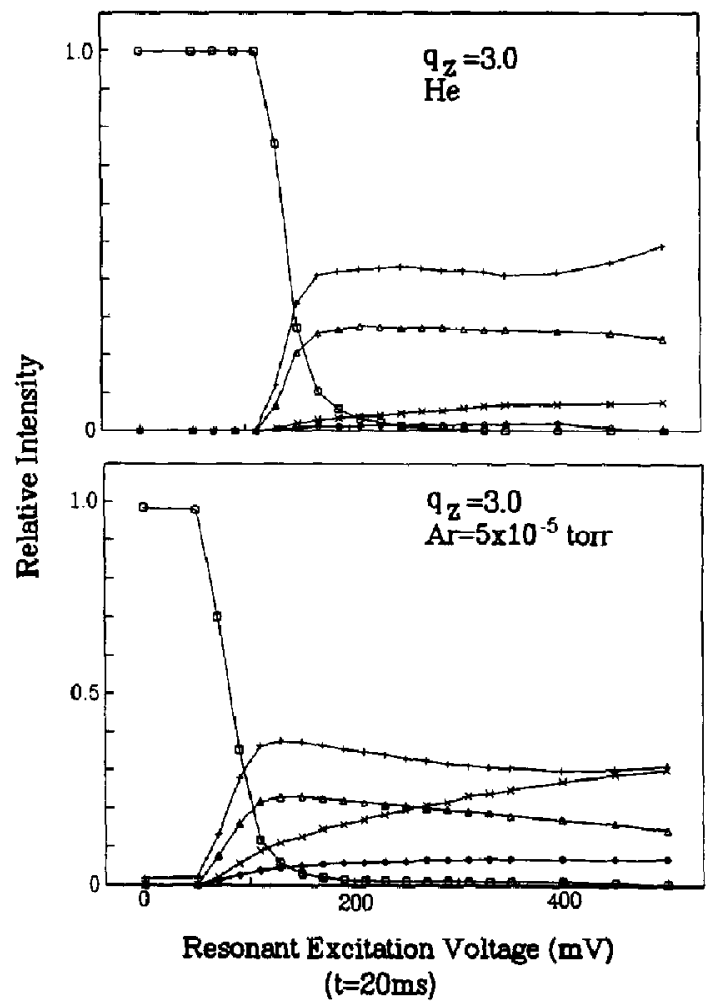

Figure 11. Plots of the relative ion intensity of the parent $(\mathrm{m} / z$ 120 from $N, N$-dimethylaniline) and product ions as a function of resonant excitation voltage for normal operation with just helium (top) and with $5.0 \times 10^{-5}$ torr or argon added to the system (bottom). $\square: m / z 120 ;+: m / z$ 118; $0: m / z 105 ; \Delta: m / z 103 ; X$ : $m / z 77$. 

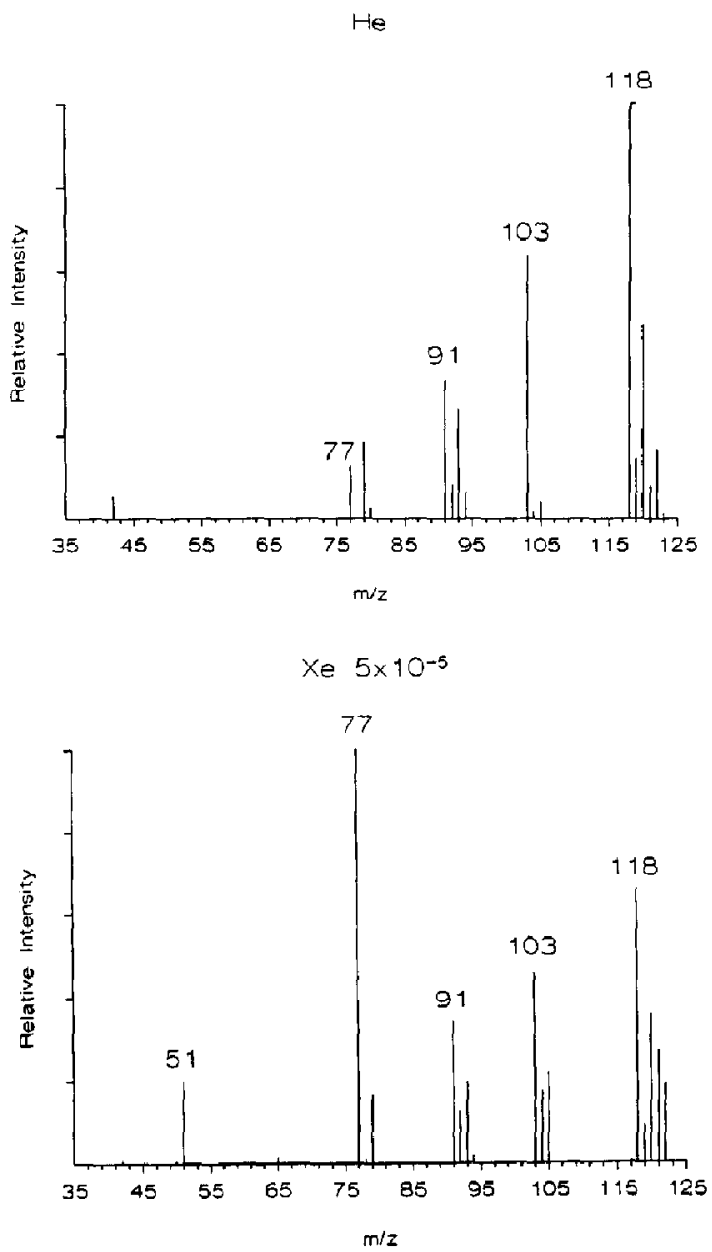

Figure 12. MS/MS of $m / z 120$ from $N, N$-dimethylaniline with (top) no xenon added and (bottom) with $5.0 \times 10^{-5}$ torr of xenon added.

more apparent if an even heavier gas than argon (e.g., xenon) is used as shown in Figure 12. The simple cleavage peaks ( $m / z 77$ and 105$)$ are greatly enhanced as are the subsequent dissociations from these ions $(m / z 77 \rightarrow 51,105 \rightarrow 104)$. (Because $m / z 77$ and 105 are not being resonantly excited, the energy to cause these ions to dissociate must be a result of the excitation of the $m / z 120$ parent ion.) Note that the relative intensity of the other ions (rearrangement processes) is essentially unchanged with respect to each other. These rearrangement ions are the product ions that are predominantly formed when the last collision before dissociation is with a helium atom.

\section{Conclusion}

The results presented here indicate that power is absorbed by reasonably excited ions from a resident excitation field according to the first term in eq 1. However, this power absorption is counterbalanced to varying extents by collisions with the bath gas (the second term in eq 1 ). Ions very quickly can reach a steady state condition in which the rate of energy loss via collision is equal to the rate energy is gained via power absorbed from the resonant excitation field. Thus, increasing the duration of resonant excitation at a given amplitude does not necessarily guarantee an increase in kinetic energy or internal energy. In CID MS/MS experiments the $q_{z}$ value of the ion being resonantly excited and the critical energy for dissociation are important to determine the relative rate of ion ejection versus dissociation. The pressure and composition of the bath gas affects the relative rates of ion ejection and dissociation. Increasing the pressure of the bath gas increases the collision rate, which decreases the rate of gain of kinetic energy of the ions. Ion dissociation becomes more competitive relative to ejection. The use of a heavier bath gas alsn increases the relative rate of ion dissociation versus ejection by two processes. A heavier bath gas increases the amount of kinetic energy that can be dissipated in a collision, which decreases the rate of ejection. One mode of dissipation of the kinetic energy is conversion to internal energy, which promotes ion dissociation.

\section{Acknowledgment}

Research sponsored by the U.S. Department of Energy, Office of Basic Energy Sciences, under contract DE-AC05-84OR21400 with Martin Marietta Energy Systems, Inc. MJC acknowledges an Oak Ridge Associated Universities Faculty Research Participation Fellowship.

\section{References}

1. Kascheres, C.; Cooks, R. G. Anal. Chim. Acta 1988, 215, 223.

2. Louris, J. N.; Cooks, R. G.; Syka, J. E. P.; Kelley, P. E; Stafford, C. C., Jr.; Todd, J. F. J. Anal. Chem. 1987, 59, 1677-1685.

3. March, R. E.; McMahon, A. W; Londry, F. A.; Alfred, R. L.; Toxid, J. F. J.; Vedel, F. Int. I. Mass Spectrom. Ion Processess 1989, 95, 119.

4. March, R. E.; McMahon, A. W.; Allinson, E. T.; Londry, F. A.; Alfred, R. L.; Todd, J. F. J.; Vedel, F. Int. J. Mass Spectrom. Ion Processes 1990, 99, 109.

5. Henis, J. M. S. In Ion-Molecule Reactions, Vol. 2; Franklin, J.L., Ed., Plenum: New York, 1972; Chap. 9.

6. Stafford, G. C., Jr.; Kelley, P. E; Syka, J. E. P.; Reynolds, W. E.; Todd, J. F. J. Int. J. Mass Spectrom. Ion Processes 1984, $60,85$.

7. Gronowska, J.; Paradisi, C.; Traldi, P.; Vettori, U. Rapid Commun. Mass Spectrom. 1990, 4, 306-313.

8. Louris, J.; Schwartz, J.; Stafford, G.; Syka, J.; Taylor, D. Proceedings of the 40th ASMS Conference on Mass Spectrometry and Alted Topics; Washington, DC, 1992; pp 1003-1004.

9. Julian, R. K., Jr.; Reiser, H. P.; Cooks, R. G. Proceedings of the 40th ASMS Conference on Mass Spectrometry and Allied Topics; Washington, DC, 1992; pp 1777-1778. 
10. Franzen, J. Int. J. Mass Spectrom. Ion Processes 1991, 106. 63-78.

11. Glish, G. L.; McLuckey, S. A.; Asano, K. G. /. Am. Soc. Mass Spectrom. 1990, 1, 166-173.

12. Yost, R. A.; Enke, C. G.; McGilvery, D. C.; Smith, D.; Morrison ${ }_{r}$ J. D. Int. J. Mass Spectrom. Ion Processes 1979, 30, 127-136

13. Reiser, H. P.; Kaiser, R. E.; Savickas, P. J.: Cooks, R. G. Int. I. Mass Spectrom. Ion Processes 1991, 106, 237-247.
14. McLuckey, S. A.; Glish, G. L.; Asano, K. G. Anal. Chim. Acta 1989, 225, 25.

15. Glish, G. L.; McLuckey, S. A.; Goeringer, D. E.; Van Berkel, G. J.; Hart, K. J. Proceedings of the 39th ASMS Conference on Mass Spectrometry and Allied Topics; Nashville, TN, 1991, pp 5.36-5.37.

16. Morand, K. L.; Cox, K. A.; Cooks, R. G. Rapid Commun. Mass Spectrom. 1992, 6, 520-523. 